\title{
Empirical Analysis of Optimal Stock Portfolio under the Background of COVID-19
}

\author{
Yifan $\mathrm{Li}^{*}{ }^{+1}$, Yu Luo ${ }^{\dagger 2}$, Yunqian $\mathrm{Zhou}^{\dagger 3}$ \\ ${ }^{1}$ School of Zhiyuan Shanghai Jiao Tong University Shanghai 200240, China \\ ${ }^{2}$ School of International Education Wuhan University of Technology Wuhan, Hubei 430070, China \\ ${ }^{3}$ School of Economics and Management Sichuan Normal University, Chengdu, Chengdu, Sichuan 610100, China \\ ${ }^{\dagger}$ These authors contributed equally.
}

\begin{abstract}
In the present market, there are various kinds of financial products and derivatives designed for customers, the most basic and universal of which are stocks. However, while understanding how much profit and risk that one stock can bring is relatively simple, it is more difficult and trickier to find out a suitable investment portfolio for customers, especially under particular circumstances. Therefore, this paper try to build a model to figure out the portfolio with the highest utility in the crown virus background. The utility is defined based on several theories on customers' behavior and takes many other factors that may play a crucial role on the extent of customer satisfaction into consideration. To simplify the model and focus on significant financial products, this article comes up with several necessary implications. After numerical computation, the result, a stock portfolio with the highest utility function for a given condition, is presented as an example to show how our model works. Finally, the paper analyzes the result obtained, provides a brief discussion on the pros and cons of our model, and eventually lists our possible future research fields.
\end{abstract}

\section{Introduction}

The COVID-19, which founded in Hubei Province in December 2019, spreads rapidly to China, even the whole world. Until April 16 2020, COVID-19 has already influenced more than 100 countries as well as regions, and the number of confirmed cases has reached 2.04 million. In financial markets, the biggest shock is that US stocks triggered four times the 'circuit breaker' just in one month. Besides, the Dow Jones Industrial Average (DJIA) decreased nearly 600 points. People also noticed that there are varying degrees of decline for both the S\&P 500 and the Nasdaq. Moreover, WTI crude fell to \$-37.63 per barrel on their May futures, and the Kuwait index had "circuit breaker," and TASI sank by $8 \%$. Those show that COVID-19 not only harms people's health but also brings a substantial adverse impact on the global economy. How to carry out the optimal portfolio of stocks under the background of the worldwide stock market crash has become a concern for investors.

Some scholars at domestic and abroad believe that investor's behavior and psychological factors have a significant impact on investment strategy, and they have tried to link some theories of behavioral economics with investment decision-making. For instance, Kahneman and Tversky proposed the prospect theory, arguing that people have the characteristics of loss aversion and are more sensitive to loss [1]; based on the prospect theory, Barberis, Mukherjee and Wang verified that this theory is not only helpful for the understanding cross-section of return, but also for both forward and backward-looking representations of stocks [2]. Besides, Fong W M pointed the value function is in direct proportionality to the investment period. At the same time, $\mathrm{M}-\mathrm{V}$ optimal asset allocation strategy shows as a conservative investment that its stocks are in inverse proportional relation to the investment period [3]. Besides, Cristinca Fulga added investors' preference into the common risk framework then proposed a better way to capture the actual behavior of loss-averse investors [4]. Plus, Berkelaar, Kouwenberg and Post $\mathrm{T}$ argued that when the evaluation period is short, the loss-averse investors have a smaller weight of stocks of the initial portfolio [5]. Some researchers, like Holger Kraft, Claus Munk and Frank Thomas Seifried, studied the effects of habits on consumption, and Abel built the utility function $[6,7]$. Phillip Cagan indicated that the wealth effect has an impact on the stock market, and Zou introduced wealth preferences into the growth theory to explain the economic growth and capital accumulation $[8$, 9]. Through studying investors' behavior, Bell, Loomes, and Sufgen presented the concept of regret avoidance, which is collective psychology of investors means that investors are unwilling to make any wrong decisions to avoid disappointment and regret $[10,11]$. Then Dodonova and Khoroshilov applied this concept to asset pricing and predicted the market's overreaction to various news; thereby, it can be used to explain the phenomenon of excessive volatility in stock yields [12]. In China, according to the nature of investor sentiment indicators, 
Zhou Liyun has confirmed the interaction effects among investor sentiment, crowded-trade behavior, and limited arbitrage on the cross-section of stock returns [13]. Moreover, YI Zhigao, MAO Ning, who selected particular indicators of investment sentiment in China's stock market, constructed and tested CICSI to measure investor emotions [14]. Furthermore, JIN Xiu, WANG Jia, GAO Ying found that the dynamic loss aversion portfolio model is better than the static portfolio model. Under different circumstances, the optimal asset allocation situation of influential loss aversion investors is also different [15]. Overall, investor behavior plays a significant part in investment decisions.

However, most of the existing studies analyze the portfolio from investors' behavior or overall market, and "Regret Evasion" does not operate much in the capital market. What is more, the majority of works focus on experiment or model studies. There are also few scholars combine with the social event timely, from the perspective of investor behavior, and having a targeted analysis on stock portfolio around a specific area. This paper sets the construction sector stocks in the Chinese market as an example and construct an optimal dynamic portfolio to study the impact of investor behavior on the investment decision-making. Overall, this research provides a reference for investors on investment choice of stock that may help investors maximize asset utility as expected. Meanwhile, it also supplies theoretical and empirical support for improving market response to emergencies.

\section{Data and Method}

\subsection{Data Selection}

In this paper, we chose data from January 2, 2020, to April 17,2020 , a total of 108 days, as the research objects for analysis, mainly considering that the time of the epidemic is less than half a year. If the weekly or monthly data were used, the data volume would be too small to influence the results of the model. Therefore, daily data is selected to improve the accuracy of model research.

\subsubsection{Stock Selection.}

This paper selects the top 10 highest profit margins stocks from January 2, 2020, to April 17, 2020, in the Chinese market's construction sector as the research object. The ten stocks are Lets Holdings GroupCO., Ltd, Zhejiang Southeast Space Frame Co., Ltd, Anhui Honglu, Steel Construction(Group) CO., Ltd, Zhejiang Yasha Decoration Co., Ltd, Ningbo Construction Co., Ltd, Hongrun Construction Group Co., Ltd, Hangxiao Steel Structure Co., Ltd, China Design Group Co., Ltd, China Communications Construction Co., Ltd, Tengda Construction Group Co., Ltd.

\subsubsection{Market Data Selection.}

This research selects the closing prices of 10 stocks, as we mentioned before, during the epidemic situation, from which the yield and standard deviation of each stock can be calculated that can meet the model requirements. There are two reasons why we choose the infrastructure sector: one is on the policy side. The resumption of all professions has been delayed as the influence of COVID-19. For infrastructure investment, the impact on it exceeded $10 \%$ in the first quarter, and the construction index also saw significant volatility, which fell $4.72 \%$ cumulatively. As the most direct method to macro-control, the government launched a series of policies to boost and promote the infrastructure investment as well as the recovery of construction companies' cash flow, thus increasing the valuation of the whole sector. Therefore, the industry of infrastructure has an excellent repair and investment opportunities; another is the shift in investor risk appetite strategy. For a long time, investors prefer high-risk preference investments such as the large technology stocks with high PE ratio and the low-priced stocks that become the popularity of the market. Nonetheless, investors realized the market risk and the severe consequence of the bursting of the high-tech bubble through the market crash happened at the end of February. As a result, some people began to look for those undervalued stocks which have slight gains and solid defense, and the many stocks of the infrastructure sector can satisfy these needs, which means this kind of stocks have advantages of investment speculation.

\subsubsection{Risk-free Interest Rate Selection}

In this paper, the interest rate of the bank of China's 2019 half-year deposit is selected as the risk-free interest rate.

\subsection{Calculation of Stock Return Rate and Standard Deviation}

In the model construction below, the return rate and the risk of investment products need to be considered. Therefore, this paper calculated the percentage of each stock during the epidemic period and the variance of the stock price during the epidemic period.

\subsubsection{Stock Returns during the Epidemic}

$$
R_{i}=\left(P_{i t}-P_{i 0}\right) / P_{i 0}
$$

Where, $R_{i}$ the yield from January 2, 2020, to April 17; $P_{i t}$ is the closing price of the $i$ th stock on April 17; And $P_{i 0}$ is the closing price of the ith stock on January 2.

\subsubsection{Standard Deviation of Stock Prices during the Epidemic Period}

$$
\begin{gathered}
\sigma_{i}=\sqrt{\frac{\sum_{j=1}^{108}\left(P_{i j}-\bar{P}\right.}{108}} \\
\overline{P_{i}}=\frac{\sum_{j=1}^{108} P_{i j}}{108}
\end{gathered}
$$

Where $\sigma_{i}$ is the standard deviation of the stock price 
and $P_{i j}$ is the closing price of the ithstock on day $\mathrm{j}$.

\subsection{The Construction of Optimal Stock Portfolios in the Context of Covid-19}

In the context of the COVID-19 outbreak, the construction industry has been dramatically impacted, and investors' investment utility may be significantly changed. To establish the best investment portfolio in this section, this paper constructs the utility function of regret avoidance investors with the help of prospect theory and the concept of regret avoidance. The top 10 stocks in the construction sector during the epidemic period (20201.2-4.2020.4.22) with no risk investment were selected for analysis, hoping to obtain the optimal stock portfolio in the construction sector that maximizes the effectiveness of investors and controls the risks within a specific range.

\subsubsection{Establishment of the Model}

The construction of the target function. The return rate of risk-free assets in the market is $r_{f}$, and the return rate of the ith stock is $\tilde{r}_{i}, i=1,2 \ldots 11$ the investment risk of each stock is $\sigma_{i}, i=1,2 \ldots 11$. The initial investment wealth owned by the investor is $W_{0}$ is paper will allocate the initial wealth between the risk-free assets in the market and the 10 stocks selected. Suppose the decisive variable, the proportion of each investment in the initial wealth is $\omega_{i}, i=1,2 \ldots 11$, the percentage of investment in risk-free assets is $\omega_{0}, \mu_{i}$ is the expected value of $\tilde{r}_{i}, i=$ $1,2 \ldots 11$

Based on the prospect theory proposed by Kahneman and Tversky and the concept of regret avoidance proposed by Bell, Loomes, and Surgen, the amount of the wealth that investors will finally get is

$$
\widetilde{W}=W_{0}\left(1+r_{f}\right)+W_{0} \sum_{i=1}^{11} \omega_{i}\left(\tilde{r}_{i}-r_{f}\right)
$$

The subjective wealth expected by the investor is

$$
E \widetilde{W}=W_{0}\left(1+r_{f}\right)+W_{0} \sum_{i=1}^{11} \omega_{i}\left(\mu_{i}-r_{f}\right)
$$

Then the utility function of the regretful evaluative investor is

$$
\begin{aligned}
\max U(\widetilde{W}, E \widetilde{W}) & \\
& =\ln (\widetilde{W})-k g[\ln (E \widetilde{W}) \\
& -\ln (\widetilde{W})]
\end{aligned}
$$

This paper sets three restricted conditions: firstly, different investors have a different expected rate of return $\alpha$ of portfolios. Secondly, investors will consider risk in the investment, the broadest acceptance of its portfolio risk is $\sigma_{\max }$, thirdly, the sum of the risk-free investment and the proportion of each stock investment is 1 , namely

$$
\left\{\begin{array}{c}
W_{0}\left(1+r_{f}\right)+W_{0} \sum_{i=1}^{11} \omega_{i}\left(\mu_{i}-r_{f}\right)=W_{0}(1+\alpha) \\
\sum_{i=1}^{11} \omega_{i} \sigma_{i} \leq \sigma_{\max } \\
\sum_{i=0}^{11} \omega_{i}=1
\end{array}\right.
$$

\subsection{Solution Algorithm}

This paper adopts the Cuckoo Search Algorithm to solve this model. The first step is to initialize the basic parameters of the algorithm, setting the number of bird nests at 11 , the dimension at $\mathrm{m}$, the number of iterations at $\mathrm{t}=0$, the maximum number of iterations at tmax, and the probability of discovery at $p_{a}$. Then initialize the nest location, based on the ROV rules, using the position relationships between the size of the component value, combining random keys to encode a bird's nest $x^{0}=$ $\left[x_{1}^{0}, x_{2}^{0}, \ldots, x_{n}^{0}\right]^{T}$, calculating values of all the bird's nest and the current optimal position of bird's nest $x_{i}^{0}(i=$ $1,2, \ldots, n)$. The third step is to start iterations, with the optimal position of bird's nest $x_{i}^{(t-1)}(t=2,3, \ldots, n)$, if the objective function value of $x_{i}^{t}$ is smaller, then $x_{i}^{(t-1)}$ will be replaced by the $x_{i}^{t}$. The new position of the bird's nest is

$$
x_{i}^{(t)}=x_{i}^{(t-1)}+\alpha S, i=1,2, \ldots, n
$$

Where, $x_{i}^{(t)}$ presents the nest position of the ithcuckoo in the tthgeneration, $\mathrm{S}$ is the walking step length, $\alpha>0$ is the step factor, $\alpha=1$ in this paper. Levy flight based on the crossing is adopted to make the nest approach to the optimal global position, and its migration formula is

$$
L(\beta)=0.01 \frac{u}{v}\left(x_{i}^{(t)}-x_{b}^{(t)}\right)
$$

Where $\quad u=t^{-\beta}, 1<\beta<3, u \sim N\left(0, \sigma_{u}^{2}\right), v \sim$ $N(0,1)$.Next, according to the discovery probability, a high-quality bird nest need to be selected with a random number of $p \in(0,1)$, which followed a uniform distribution, as the host's possibility of discovering foreign eggs, and compared with the fixed discovery probability $p_{a}$. Delete the nest position with a probability higher than $p_{a}$ in $x_{i}^{(t)}$, and choose the nest position with a probability less than $p_{a}$. Calculated objective function value of each nest and record the current optimal nest position after the position is moved. Then compare the minimum value of the objective function of $x_{i}^{(t)}$ and $x_{i}^{(t-1)}$, if the minimum value of the current generation is less than the original minimum value, the minimum value of the current generation will be taken as the corresponding objective function value of the new nest position to obtain a new set of better positions. Finally, find the position obtained in the previous step and determine whether the number of 
iteration reaches the maximum number of iterations. If so, output the global optimal scheduling value and the corresponding scheduling solution. If not, return to continue the iteration.

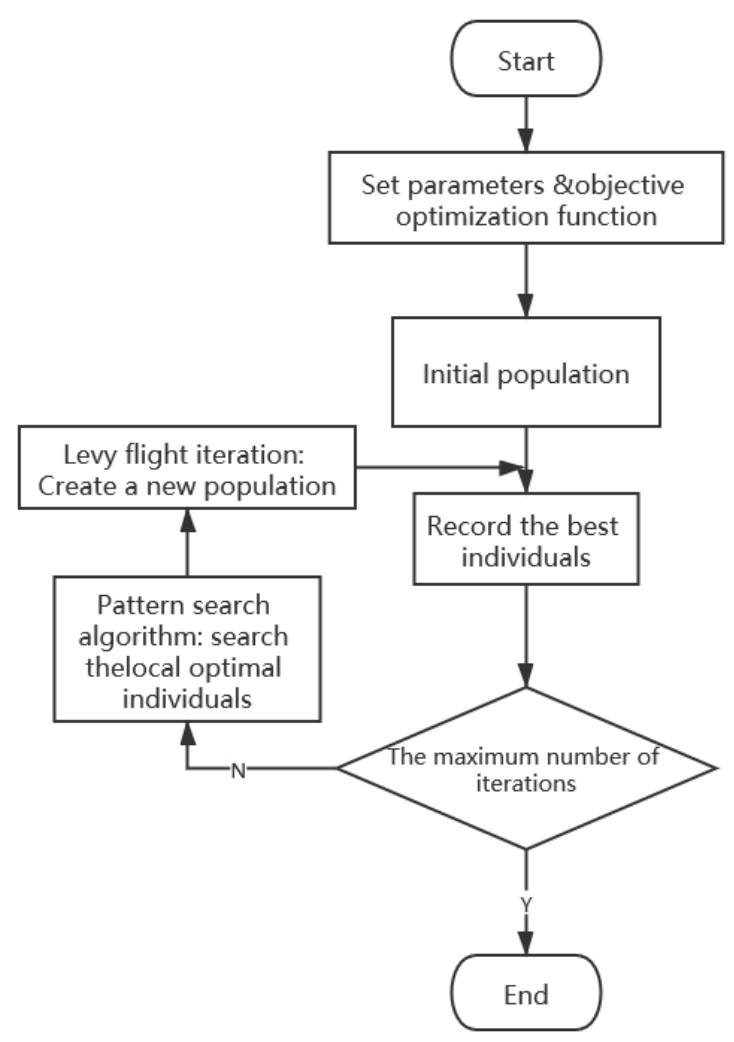

Fig1. The process of Cuckoo Search

\section{Results and Discussion}

\subsection{Data selection}

We choose four companies' price trend as examples:

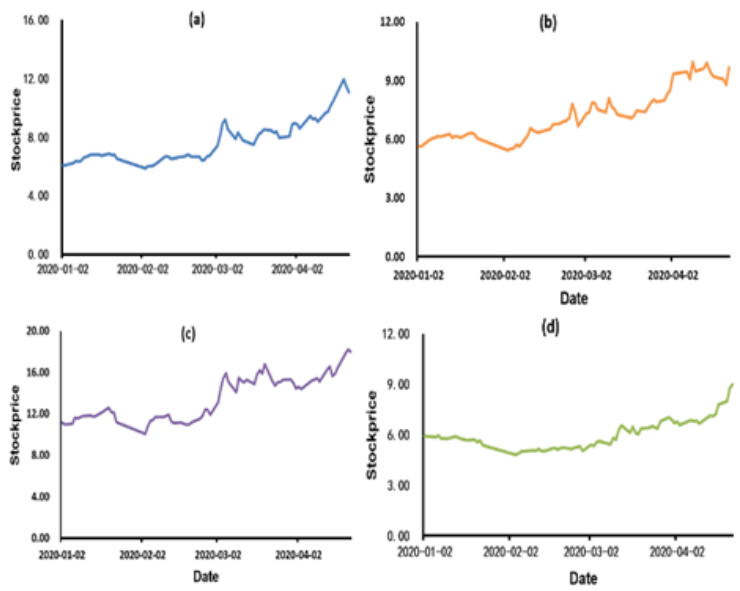

Fig2. (a) Price trend of Lets Holdings GroupCO.,Ltd; (b) Price trend of Zhejiang Southeast Space Frame Co., Ltd; (c) Price trend of Anhui Honglu Steel Construction(Group) CO., Ltd (d) Price trend of Zhejiang Yasha Decoration

$$
\text { Co., Ltd }
$$

\subsection{Result}

This paper adopted the cuckoo algorithm to solve the optimal stock portfolio model under the background of COVID-19. Suppose investors expected return rate is $5 \%$, for example, set parameter $\theta=2$. The regret aversion function based on the theory of Bell turns into a quadratic function. Under the influence of the COVID-19, investors tend to be more cautious and will be less affected by emotion, so this paper takes a small regret degree parameter $=0.5$, and the most significant risk for investors is 0.1 . As a result, the proportions of investors' wealth among the top 10 stocks in the construction market and risk-free investment are shown in Table 1.

Table1. The results of optimal portfolios

\begin{tabular}{cc}
\hline Company name & Investment ratio \\
\hline Lets Holdings GroupCO., Ltd & $0.18 \%$ \\
Zhejiang Southeast Space Frame Co., Ltd & $0.35 \%$ \\
Anhui Honglu Steel Construction(Group) & $1.2 \%$ \\
CO., Ltd & $2.15 \%$ \\
Zhejiang Yasha Decoration Co., Ltd & $22.53 \%$ \\
Ningbo Construction Co., Ltd & $2.44 \%$ \\
Hongrun Construction Group Co., Ltd & $31.38 \%$ \\
Hangxiao Steel Structure Co., Ltd & $2.62 \%$ \\
China Design Group Co., Ltd & $0.4 \%$ \\
China Communications Construction Co., & $0.34 \%$ \\
Lengda Construction Group Co., Ltd. & $36.39 \%$ \\
Risk-free investment
\end{tabular}

We can see from Table 1 that, with the yield equal to $5 \%$ and lower risk tolerance, the risk-free investment's proportion is the largest in the optimal portfolio. The following are those stocks with lower risk, which presents that during the outbreak of COVID - 19, those investors affected less by the emotions prefer those less risky assets, the return on investment is not the primary consideration for them.

In the calculation process, we choose several different time steps to ensure an investment portfolio free of influence from the original nest position. The sum of the investment ratio is very close to 1 , as we expected.

\section{4 conclusion}

The result above clearly shows that in this condition, the investment is mainly distributed on three parts: Risk-free investment, Hangxiao Steel Structure Co., Ltd, and Ningbo Construction Co., Ltd. Following is a brief analysis of the reason why these three investments are chosen in the portfolio. According to our assumption, the risk-free investment, which is assumed to be a six-month time deposit return rate, is not related to any of the ten stocks, so for clients who would like to restrict the risk of their investments, it is a safe choice despite its low return rate. The historical data indicate that Hangxiao Steel Structure Co., Ltd, and Ningbo Construction Co., Ltd have relatively small variance and comparatively high profit, so they can fulfill the limitations on the risk while 
maintaining a competitive return rate. On the contrary, stocks with exceptionally low return rates or manifest fluctuations, such as Lets Holdings GroupCO., Ltd, are not selected. In this way, the correctness of the model for designing a stock portfolio is confirmed initially. Particularly, it is suitable for users who are able to quantify their expectations on the overall return rate and maximum risk. It may also serve as a useful reference for investors during this unstable economic situation caused by COVID-19.

With the rapid development of the building industry in China, its intrinsic value is continuously growing under the help of the government. And because the demand of infrastructure is still steadily increasing, the inherent value of the building stocks is to some extent safer than that of many high technology industries, such as IT. Apart from that, our result reflects the importance of investment diversification. By distributing the investment to several stocks, the risk could be significantly reduced. As a generalization, it is a wise choice to invest in stocks from different domains since they may have a smaller relation coefficient. Thus, the total risk of the stock portfolio would diminish.

Admittedly, there are some limitations to our research, which could be investigated later. To start with, this article only considers ten stocks with the best performance in the building industry, and the portfolio return rate could be enhanced if the portfolio includes stocks in other domains according to the generalization mentioned above. Besides, the impact of several parameters, for example, the regretavoiding coefficient $\theta$, on the result of our model is not thoroughly tested or investigated. Finally, the number of stocks this article chooses may not be enough to find out the most desirable portfolio for the clients. In fact, the model will provide particularly risky choices if it receives regulations that are out of range, for example, an expected return rate, which is significantly higher than that of any stock considered. Future studies will expand the model's applicability and try to find a more exact relationship between the used parameters and customers' behavior.

\section{References}

1. Daniel Kahneman, and Amos Tversky, "Prospect Theory: an Analysis of Decision under Risk," Econometrica, vol. 47, No. 2, March 1979, pp. 263291.

2. Barberis, Nicholas, A. Mukherjee, and B. Wang, "Prospect Theory and Stock Returns: An Empirical Test," Review of Financial Studies, vol. 29, 2016, pp. 3068-3107.

3. Fong W M, "Time diversification under loss aversion: a bootstrap analysis," Applied Economics, vol. 45, 2013, pp. 605-610.

4. Fulga C, "Portfolio Optimization under Loss Aversion," European Journal of Operational Research, vol. 251, 2016, pp. 310-322.

5. Berkelaar A B, Kouwenberg R, and Post T, "Optimal Portfolio Choice under Loss Aversion," Review of Economics and Statistics, vol. 86, 2004, pp. 973-987.
6. Holger Kraft, Claus Munk, and Frank Thomas Seifried, "Consumption habits and humps," Economic Theory, vol. 64, 2017, pp. 305-330.

7. Abel, "A. B. Asset prices under habit formation and catching up with the joneses," American Economic Review Papers and Proceeding, vol. 80,1990, pp. 3842.

8. Cagan P, "The 1987 Stock Market Crash and the Wealth Effect," Analyzing Modern Business Cycles, 2019.

9. Zou, and H.-F, “The spirit of capitalism, social status. money and accumulation," Journal of Economics, vol. 68, No. 3, 1998, pp. 219-233.

10. Bell, and David E, "Risk Premiums for Decision Regret," Management Science, vol.29, 1983, pp. 1156-1166.

11. Loomes G, and Sufgen R, "Regret theory: an alternative theory of rational choice under uncertainty," the Economic Journal, vol. 92, 1982, pp. 805-824.

12. Dodonova A, and Khoroshilov Y, "The spirit of capitalism, social status. money and accumulation," SSRN Electronic Journal, 2005.

13. Zhou Liyun, and C. Yang, "Investor sentiment, investor crowded-trade behavior, and limited arbitrage in the cross section of stock returns," Empirical Economics ,2019.

14. YI Zhigao, and MAO Ning, "Research on the Measurement of Investor Sentiment in Chinese Stock Market: the CICSI's Construction ," Journal of Financial Research, vol. 000, 2009, pp. 174-184.

15. JIN Xiu, WANG Jia, and GAO Ying, "Optimal Asset Allocation Based on Dynamic Loss Aversion Portfolio Model and Empirical Research," Chinese Journal of Management Science, vol. 022, 2014, pp. 16-23. 